\title{
Lactobacillus crispatus DSM25988 as novel bioactive agent to co-aggregate Streptococcus pyogenes and to exclude it by binding to human cells
}

\author{
A. Reiprich, L. Skalden, A. Raab, N. Bolotina and C. Lang" \\ Belano medical AG, Neuendorfstraße 19, 16761 Hennigsdorf, Germany; christine.lang@belanomedical.com
}

Received: 22 July 2021 / Accepted: 18 December 2021

(c) 2022 Wageningen Academic Publishers

OPEN ACCESS (1) (9) RESEARCH ARTICLE

\begin{abstract}
Streptococcus pyogenes, a group A streptococcus, is the major bacterial pathogen responsible for acute bacterial infection of the human oropharynx and the causative agent of scarlet fever. Estimates of the global burden of S. pyogenes related diseases revealed 616 million cases of pharyngitis, and at least 517,000 deaths due to severe invasive diseases and sequelae. Here we describe Lactobacillus crispatus DSM25988 that was identified among hundreds of Lactobacillus strains (referring to all organisms that were classified as Lactobacillaceae until 2020) showing ability to prevent adhesion of S. pyogenes to Detroit 562 cells, and to exhibit a masking and co-aggregating effect on S. pyogenes in vitro. L. crispatus DSM25988 also inhibits invasion of cultured human epithelial pharyngeal cells by $S$. pyogenes. Competitive binding to fibronectin might be involved in the inhibition process. Antiviral activity of the $L$. crispatus DSM25988 cells were identified in an in vitro cell model demonstrating that $L$. crispatus effectively excludes viruses from epithelial cells using SARS-CoV2 proteins as a model. This finding points to the potential of DSM25988 to protect cells from virus infection. Biological activity is retained in heat treated cells. The heat-treated Lactobacillus strain was further developed into functional throat lozenges, wherein its biological activity is stably maintained in the formulation. Lozenges containing L. crispatus DSM25988 underwent testing in an uncontrolled, prospective user study in 44 subjects with symptoms of sore throat for a period of up to 14 days. The study data shows promising safety and efficacy of the medical device when used against symptoms of sore throat like scratchy feeling, hoarse voice and swallowing pain.
\end{abstract}

Keywords: group A streptococcus, SARS-CoV2, lactic acid bacteria, throat lozenges, user study

\section{Introduction}

Streptococcus pyogenes is a human bacterial pathogen with a tremendous virulence potential. It primarily colonises the upper respiratory pharyngeal mucosa (Walker et al., 2014). Interactions with humans range from asymptomatic carriage over mild and superficial infections of mucosal membranes and skin up to systemic purulent toxicinvasive disease manifestations. S. pyogenes is the major bacterial pathogen responsible for acute infection of the human oropharynx and tonsils, and it is the causative agent of scarlet fever. S. pyogenes pharyngitis is commonly associated with sore throat, pain on swallowing, and fever. Estimates of the global burden of S. pyogenes related diseases revealed 616 million cases of pharyngitis and at least 517,000 deaths due to severe invasive diseases and sequelae (Carapetis et al., 2005). Although pharyngitis in the majority of cases primarily is caused by viral infections, S. pyogenes is responsible for $5-15 \%$ of sore throat visits in adults and $20-30 \%$ in children. The incidence peaks in children 5-15 years of age, and, in temperate climates, pharyngitis usually occurs in the winter and early spring. The risk of acute pharyngitis due to S. pyogenes among adults is higher for parents of school-age children and for those whose occupation brings them into close association with children (Lindbaek et al., 2004). Asymptomatic carriage of $S$. pyogenes has been frequently noted among household contacts of patients with $S$. pyogenes pharyngitis. Moreover, S. pyogenes carriage by symptom-free health care workers has also been made responsible for nosocomial outbreaks (Danzmann et al., 2013). In symptom-free children aged 2-4 years, carriage of 100 to $10,000 \mathrm{cfu} / \mathrm{ml}$ 
S. pyogenes per nasopharyngeal swab has been detected (Thors et al., 2016).

Much research has been done to reveal molecular interactions between S. pyogenes and its human host. Several cell surface proteins of $S$. pyogenes have been documented as being involved in pharyngeal adherence and colonisation during infection, including lipoteichoic acid, M-protein and collagenlike surface proteins Scl1 and Scl2. The adhesion proteins, and specifically the Scl proteins have been implicated in binding to a wide variety of factors on the surface of host pharyngeal cells, including fibronectin, laminin and collagen (Courtney et al., 1986; Ellison et al., 2020). A number of antibiotics have been shown to be effective in treating $S$. pyogenes pharyngitis. These include penicillin and its congeners (e.g. ampicillin and amoxicillin), as well as numerous cephalosporins, macrolides, and clindamycin (Shulman et al., 2012). However, the use of antibiotics heavily disrupts the ecology of the human microbiota not only of the pharynx, but also of the gut, skin and other mucosal tissues. Dysbiosis of the microbiota has been associated with a large number of health problems and causally implicated in metabolic, immunological, and developmental disorders, as well as susceptibility to development of infectious diseases. The adverse effects of antibiotics on microbial succession, diversity, and resistance can last long past application, even for years (Langdon et al., 2016).

Over the past decades, lactic acid bacteria have aroused interest for their use in prevention and treatment of multiple diseases owing to the health beneficial (probiotic) properties of some strains (Buckley et al., 2018; Holz et al., 2013, 2015). Mechanisms of action may include enhanced mucosal barrier function, direct antagonism with pathogens, inhibition of bacterial adherence and invasion capacity in the intestinal epithelium, boosting of the immune system and influence on the central nervous system (Shenderov et al., 2020; Stavropoulou and Bezirtzoglou, 2020). Probiotics have also been considered an alternative to antibiotics (Kim et al., 2015; Reid and Friendship, 2002). Lactobacillus crispatus is a commensal gram-positive lactic acid bacterium inhabiting the human vaginal and intestinal mucosa, and several strains are highly adhesive to different types of epithelial cells (He et al., 2020). Phylogenetically, L. crispatus is closely related to Lactobacillus acidophilus and Lactobacillus helveticus, two of the most commonly used probiotics in food industry (You and Kim, 2020). L. crispatus has a long history of human consumption and has been identified in starter cultures for rye sourdough, in cheese, wine, sauerkraut and in whisky (Beganovic et al., 2014; Henri-Dubernet et al., 2008; Kačániová et al., 2012; Meroth et al., 2003; Van Beek and Priest, 2000). L. crispatus is classified as biosafety level 1 , is Generally Regarded As Safe (GRAS), and is approved as a 'Qualified Presumption of Safety' (QPS) microorganism, which conforms with the safety requirements for a food or feed additive of the European Food Safety Authority
(EFSA) (Andreoletti et al., 2008). Epidemiologic studies reveal a strong positive correlation between the presence of L. crispatus in the vaginal microbiota and reduced urogenital infection risk to bacterial, fungal and viral pathogens (Ngugi et al., 2011; Wang et al., 2019). Further, there is preclinical and clinical experimental evidence of $L$. crispatus reducing the urogenital and gastrointestinal infection risk towards multiple fungal, bacterial and viral pathogens (Horie et al., 2002; Mousavi et al., 2018; Parolin et al., 2018; Stapleton et al., 2011). Surface layer (S-layer) proteins, such as SlpA or CbsA are considered relevant for the antipathogenic activity of $L$. crispatus. These proteins support adherence of $L$. crispatus to epithelial cells of the cervix, thus sterically competing with, excluding or displacing pathogens from the mucosal surface (Abramov et al., 2014; Chen et al., 2007). Strain-specific adhesins from different L. crispatus strains could be identified as mucus-binding proteins. It appears that $L$. crispatus via its adhesins is able to interfere with fibronectin binding of pathogens to epithelial cells, thus reducing the colonisation of the pathogen (Ojala et al., 2014). Moreover, L. crispatus has been described to adhere to fibronectin, laminin and to collagens I, IV and V, thus competing with pathogens for these proteins (Antikainen et al., 2002). Besides this steric shielding effect, $L$. crispatus is also able to directly interact with pathogens on the basis of direct cell-cell contacts (Mousavi et al., 2018; Parolin et al., 2018).

Here, we describe the characterisation of heat-treated L. crispatus strain DSM25988 selected from a large lactic acid bacteria strain collection as a suitable lactic acid bacteria strain for the prevention of S. pyogenes adhesion to laropharyngeal epithelial cells, as well as a bioactive for the prevention and treatment of $S$. pyogenes-caused sore throat with its clinical application as a lozenge.

\section{Materials and methods}

\section{Bacterial strains}

Lactobacillus species strains from a proprietary strain collection were grown at laboratory scale and exposed to a systematic preclinical screening program as viable bacteria or after heat-treatment (OASSYS screening system; Organobalance GmbH, Berlin, Germany). The final hit was identified as L. crispatus by $16 \mathrm{~S}$ rDNA sequence analysis. For whole genomic sequencing, DNA of L. crispatus DSM25988 was extracted (Leibniz Institute DSMZ GmbH, Braunschweig, Germany) and sequenced by Next Generation sequencing using PacBio RS technology (Rhoads and $\mathrm{Au}, 2015$ ) followed by assembly of long high-quality reads to obtain the whole genome sequence (Eurofins Genomics GmbH, Ebersberg, Germany). Specieslevel taxonomic identification was performed (BaseClear BV, Leiden, the Netherlands). Potential antibiotic resistance genes and virulence factors were searched for by comparing 
the aligned and annotated full genome sequence to the Comprehensive Antibiotic Resistance Database (CARD) (McMaster University, Ontario, Canada) and Virulence Factor Database (VFDB) (NHC Key Laboratory of Systems Biology of Pathogens, Institute of Pathogen Biology, Beijing, China PR) databases in October 2019 (BaseClear BV). At lab scale, L. crispatus DSM 25988 was grown in MRS medium (De Man et al., 1960) at $37^{\circ} \mathrm{C}$ under anaerobic conditions if not described otherwise. L. crispatus DSM25988 was industrially manufactured by fermentation in yeast extract media at $37^{\circ} \mathrm{C}$, followed by heat treatment, cell separation and lyophilisation using maize starch for stabilisation (Lactosan GmbH \& Co KG, Kapfenberg, Austria). S. pyogenes DSM11728 and S. pyogenes DSM20565 were obtained from the German Collection of Microorganisms and Cell Cultures GmbH (DSMZ, Braunschweig, Germany). Cells were grown in Todd-Hewitt-broth (Ellner et al., 1966) at $37{ }^{\circ} \mathrm{C}$, in a microaerobic atmosphere or at $5 \% \mathrm{CO}_{2}$ with $96 \%$ humidity if not described otherwise.

\section{Co-aggregation}

The test for co-aggregation activity of lactobacilli with S. pyogenes DSM11728 was adapted from Lang et al. (2010) and Holz et al. (2015) with the difference that the optical density (OD $600 \mathrm{~nm}$ ) was adjusted to 2 (S. pyogenes), and to 1 (Lactobacillus species). S. pyogenes cells were stained with Vybrant ${ }^{\oplus}$ CFDA SE (Fisher Scientific GmbH, Hagen, Germany) adapted from Lang et al. (2010). For coaggregation testing of salvans lozenges, one verum lozenge containing L. crispatus and one lozenge without lyophilised heat-treated L. crispatus (placebo), were dissolved in phosphate buffered saline (PBS) and subjected to the assay with unstained S. pyogenes cells.

\section{Biofilm binding of Lactobacillus cells}

In order to quantify binding of lactobacilli to $S$. pyogenes DSM11728 the optical density $\left(\mathrm{OD}_{600}\right)$ of washed $S$. pyogenes cells in PBS was adjusted to 1 , transferred to 96 well microtiter plates for an overnight incubation at $37^{\circ} \mathrm{C}$ to form a biofilm (Stepanović et al., 2000). Lactobacillus cells grown overnight were stained with Vybrant CFDA SE. Aliquots of stained Lactobacillus cells were co-incubated with the $S$. pyogenes biofilm for $1 \mathrm{~h}$ and washed three times with PBS to remove unbound or loosely bound cells. Fluorescence was measured before and after incubation at $494 \mathrm{~nm} / 512 \mathrm{~nm}$ and after each washing step with a Mithras Lb 940 multiplate reader (Berthold Technologies $\mathrm{GmbH}$ \& Co.KG, Bad Wildbad, Germany).

\section{Pharyngeal epithelial cell line invasion inhibition assay}

Knowledge of adherence assays (Plant et al., 2006) led to the approach of investigating the invasion inhibition of S. pyogenes by L. crispatus. Human pharynx carcinoma epithelial cell line Detroit-562 cells were grown in supplemented Minimal Essential Medium (MEM) with Earle's salts at $37^{\circ} \mathrm{C}, 5 \% \mathrm{CO}_{2}, 95 \%$ humidity, plated in 24 well cell culture plates and cultivated again under identical conditions for $24 \mathrm{~h}$. Non-adhering cells were removed. Washed bacterial cells were resuspended in MEM medium and the $\mathrm{OD}_{600}$ was adjusted (S. pyogenes DSM20565 OD 0.2 and L. crispatus OD 1). L. crispatus suspension was pre-incubated with adhering Detroit 562 cells for $30 \mathrm{~min}$ at $37{ }^{\circ} \mathrm{C}, 5 \% \mathrm{CO}_{2}, 95 \%$ humidity. S. pyogenes was added, and the cell culture plates were incubated again at $37{ }^{\circ} \mathrm{C}$, $5 \% \mathrm{CO}_{2}$, 95\% humidity for $30 \mathrm{~min}$. Detroit 562 cells were then detached from the culture plates by adding cold water and placing the culture plate on an orbital shaker. Cells were suspended in PBS, plated onto neomycin blood agar and incubated at $37^{\circ} \mathrm{C}$ under microaerophilic conditions. S. pyogenes colony counts were determined.

\section{Biofilm reducing assay}

The MBEC Assay ${ }^{\circledR}$ Biofilm Inoculator (Innovotech Inc., Edmonton, Canada) was used for biofilm density formation (Ceri et al., 1999; Harrison et al., 2010). Biofilm formation was tested with and without precoating of the polystyrene pegs with saliva. Pooled saliva from at least three human donors (Lee BioSolutions Inc., Maryland Heights, MO, USA) was sterile filtrated on the day of use to remove any microorganisms from the saliva. For saliva precoating, the peg lid was inserted into a microtiter plate with saliva in the respective microwells, and incubated at $37^{\circ} \mathrm{C}$ for $1 \mathrm{~h}$. Trypticase soy yeast extract medium (TSY), saliva and a 50:50 mixture of TSY and saliva were compared as growth media for establishing $S$. pyogenes biofilms. S. pyogenes was grown in TSY at $37^{\circ} \mathrm{C}$. In case of pre-treatment of S. pyogenes with Lactobacillus cells, S. pyogenes culture was incubated with lyophilised heat-treated manufactured L. crispatus ( $2 \mathrm{mg} / \mathrm{ml}$ final concentration) for $20 \mathrm{~min}$ with shaking at $120 \mathrm{rpm}$, then allowed to settle for $30 \mathrm{~min}$. The supernatant and the untreated S. pyogenes culture were used for the biofilm assay on pegs and binding was analysed by determination of cfu/peg. In each case the untreated S. pyogenes culture was compared to the pre-treated $S$. pyogenes culture to determine the effect of $L$. crispatus.

\section{Fibronectin binding assay}

Fibronectin-coated 96 well microtiter-plates (Corning ${ }^{\circledR}$ BioCoat $^{\mathrm{Tm}}$ Fibronectin 96-well plates; Corning Inc, Corning, NY, USA) were adjusted to room temperature to investigate binding to immobilised fibronectin (Courtney et al., 1986). Samples were applied and measured photometrically at wavelengths of $485 \mathrm{~nm}$ excitation and $528 \mathrm{~nm}$ emission (BioTek Instruments Inc., Winoosky, VT, USA). Cells were harvested and resuspended in $\mathrm{PBS}, \mathrm{OD}_{600}$ was adjusted. Samples of the lyophilised heat-treated manufacturing batches of $L$. crispatus were resuspended in PBS. Samples 
were stained with CFDA SE (Fisher Scientific GmbH, Hagen, Germany or Biotium, Fremont, USA). PBS and maize starch samples were treated with CFDA in the same way as controls. For competitive binding assays the washed S. pyogenes DSM20565 was stained with CFDA SE (Biolegend, San Diego, CA, USA). Lyophilised heat-treated L. crispatus was suspended in PBS incubated at room temperature. Efficiency of reduced S. pyogenes fibronectin binding was calculated as relative binding inhibition in percent to the control S. pyogenes alone.

\section{SARS-CoV2 neutralisation assay}

SARS-CoV2 microneutralisation assay was performed at IBT Bioservices (Rockville, MD, USA) Manufactured L. crispatus (heat-treated, lyophilised) was suspended in PBS, pH was adjusted to 7.4. Vero cells seeded one day prior in black 96-well plates were incubated overnight. Two-fold serial dilutions ranging from 40 to $0.3 \mathrm{mg} / \mathrm{ml}$ were prepared in triplicate. The dilutions were then incubated with recombinant rVSV-SARS-CoV2 Spike for $1 \mathrm{~h}$ at $37^{\circ} \mathrm{C}$. After removing cell culture medium from the 96 -well plates, the $L$. crispatus/virus mixtures were added to the Vero cells, and incubated for $24 \mathrm{~h}$ at $37^{\circ} \mathrm{C}$. Infection efficiency was measured by quantifying luciferase fluorescence; virus only and Vero cells only wells were added to calculate the $50 \%$ inhibition concentration $\left(\mathrm{IC}_{50}\right)$ using the XLFit dose response model.

\section{Cytotoxicity assay}

Cytotoxicity assay was performed at IBT Bioservices. Heattreated, lyophilised L. crispatus was suspended in PBS, $\mathrm{pH}$ was adjusted to 7.4. Vero cells seeded one day prior in black 96-well plates were incubated overnight. Twofold serial dilutions ranging from 40 to $0.3 \mathrm{mg} / \mathrm{ml}$ were prepared in triplicate. L. crispatus dilutions were incubated for $1 \mathrm{~h}$ at $37^{\circ} \mathrm{C}$ and, after removing cell culture medium from the 96-well plates, were added to the Vero cells and incubated for $24 \mathrm{~h}$ at $37{ }^{\circ} \mathrm{C}$. Cells only and medium only wells were also added. After incubation, cells were lysed for evaluation of the ATP content using the CellTiter-Glo ${ }^{\circ}$ kit (Promega, Madison, WI, USA). Luciferase luminescence in relative light unit (RLU) was read and $50 \%$ cytotoxicity concentration $\left(\mathrm{CC}_{50}\right)$ was calculated using the XLFit dose response model.

\section{Preparation of salvans lozenges}

Lozenges ('salvans ${ }^{\bullet}$ Halspastillen') were manufactured using isomalt, marshmallow root extract, natural flavours, natural colorants and L. crispatus salvans (Dr. C. Soldan GmbH, Adelsdorf, Germany) and packaged in aluminium/PVC blisters (Captagel Pharma GmbH, Besigheim-Ottmarsheim, Germany). The product is indicated for the prevention and relief of sore throat symptoms in patients above 6 years of age at risk of laropharyngeal infection caused by S. pyogenes, and certified as a substance-based, risk-class I medical device under GMP- and EN ISO 13485:2016 compliant manufacturing conditions (Nutrin $\mathrm{GmbH}$, Hallerndorf, Germany). Conformity was evaluated and confirmed by the manufacturer on the basis of the manufacturing validation batch.

\section{Salvans lozenges study}

A social-media based, uncontrolled observational post market user follow-up study was launched in order to generate data on user acceptability, product safety and performance regarding the relief of sore throat symptoms in subjects prone to bacterial throat infection. Interested recipients were informed about the required actions and purpose of the study and data protection rights according to the European General Data Protection Regulation (EU) 2016/679 (GDPR; EC, 2016). Subjects willing to participate were asked to explicitly consent in writing to study participation and the collection, storage and use of their data for the purpose of the study. Via an electronic online questionnaire, subjects were requested to provide data on the frequency and duration of sore throat episodes over the last 5 years, the specification of common cold symptoms during these episodes, diagnosis of streptococcal angina by a general practitioner during this time, and prescription of antibiotics. Inclusion criteria were at least 2 annual sore throat episodes over the past 5 years, the occurrence of symptoms of bacterial throat infection or diagnosis of streptococcal angina by a general practitioner and a history of prescription of antibiotics. Exclusion criteria was the current use of antibiotics. The selected cohort of participants was provided with one package of lozenges (24 lozenges) and asked to complete an electronic baseline-questionnaire (baseline), as soon as they suffer from symptoms of sore throat, and another questionnaire after symptoms disappeared (follow-up). The data from the baseline and follow-up questionnaires was exported from the web application in MicrosoftExcel format, merged in Microsoft Excel (Microsoft, Redmond, WA, USA), quality checked and exported in csl-format. Descriptive data analysis was performed using the freeware PSPP. The following data were collected: demographics (age, gender), concomitant treatments, date of first lozenge application, occurrence of the common cold symptoms (irritated throat sensation, oropharyngeal redness, swallowing pain, hoarseness, running nose, cough (dry, productive), increased body temperature, fever, body aches, headache, ear pain, swollen lymph nodes, purulence, duration of symptoms at start of lozenge application, Visual Analog Scale (VAS) (0-10) assessment of throat pain, burning, swallowing pain, sensation of throat dryness, hoarseness, sensation of throat irritation, feeling of 'lump in the throat', inflammation, swelling, tight sensation, VAS (0-10) assessment of improvement of throat pain after 
one lozenge (and speed of improvement), improvement of throat pain after one day of lozenge application (and number of lozenges).

\section{Results}

\section{Selection of Lactobacillus strains co-aggregating with Streptococcus pyogenes}

In the initial mass screening program, 798 lactobacilli strains ('lactobacilli' and Lactobacillus spec. designating all organisms that were classified as Lactobacillaceae until 2020) were evaluated for their binding capacity and binding strength to $S$. pyogenes. A subset of 89 (11.1\%) lactobacilli strains showed medium to very strong binding. In follow-up investigations, the subset of 89 Lactobacillus strains was screened for their ability to co-aggregate with $S$. pyogenes in aqueous suspension. Thirty strains were identified to co-aggregate the $S$. pyogenes strain. Among these, a subset of five candidate strains was further analysed regarding efficiency of co-aggregation, fibronectin binding and binding to a pharyngeal epithelial cell line. Moreover, the candidates were analysed by 16 s rRNA gene sequencing and whole genome analysis. The finally selected candidate was identified as L. crispatus (deposited under DSM25988). No transmissible antibiotic resistance genes or virulence factors were found when matching the genome of this strain to the CARD and VFDB databases. Living as well as heat-treated L. crispatus DSM 25988 cells were able to form large co-aggregates with S. pyogenes (Figure 1), involving multiple streptococci adhering to one Lactobacillus cell (microscopic analysis, data not shown).

\section{Lactobacilli reduce adherence of Streptococcus pyogenes to human epithelial cells}

In an adherence assay, we investigated if the pre-selected lactobacilli interfered with $S$. pyogenes binding to human epithelial host cells. Lactobacilli indeed reduced binding of $S$. pyogenes in a competition assay, though in different degrees (Figure 2). L. crispatus DSM25988 (strain 3 in Figure 2) reduced binding of $S$. pyogenes by up to $22 \%$.

Interference of $L$. crispatus (heat-treated lyophilised cells) with biofilm formation of S. pyogenes was analysed on salivacoated or uncoated polystyrene pegs. Pre-incubation with L. crispatus DSM25988 significantly reduced S. pyogenes biofilm density by 1.35 and $0.85 \log$ on the saliva-coating and uncoated polystyrene plates, respectively, indicating that Lactobacillus cells reduce biofilm formation and prevent binding of the pathogen.

\section{Lactobacillus crispatus efficiently binds to fibronectin}

To further characterise and understand the observed adherence inhibition of S. pyogenes by L. crispatus on human epithelial cells, we analysed fibronectin binding activities.

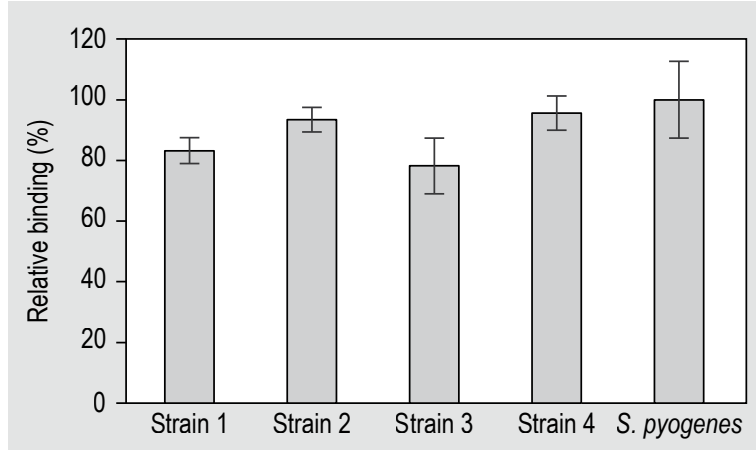

Figure 2. Invasion inhibition assay of selected lactobacilli against Streptococcus pyogenes on Detroit-562 cells. Lactobacilli from the screening cohort (strain 1-4) were preincubated for $30 \mathrm{~min}$ to human pharynx carcinoma epithelial cell line Detroit 562, S. pyogenes was added and incubated for $30 \mathrm{~min}$. The control (labelled 'S. pyogenes') is a human cell layer pre-incubated with phosphate buffered saline before incubation with $S$. pyogenes.

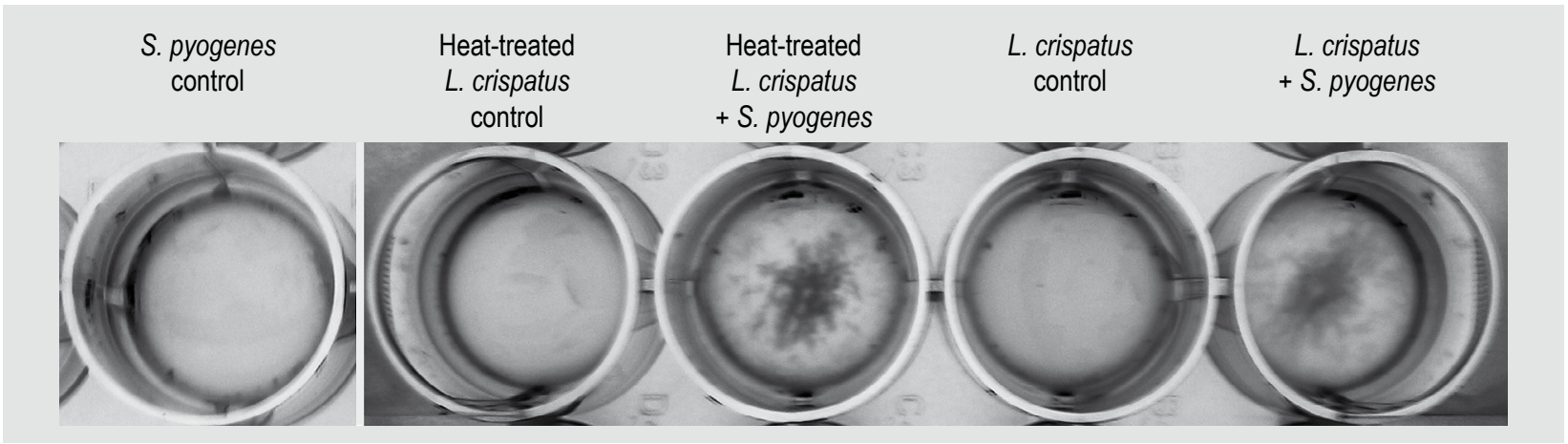

Figure 1. Co-aggregation of living and heat-treated Lactobacillus crispatus DSM25988 with Streptococcus pyogenes DSM11728. The results are shown compared to negative control (L. crispatus). S. pyogenes alone does not show any precipitation. Living and heat-treated $L$. crispatus DSM25988 induced comparable degrees of co-aggregate formation with $S$. pyogenes, and comparable in vitro efficacy in reducing planktonic $S$. pyogenes from the supernatant. 
L. crispatus DSM25988 and heat-treated lyophilised L. crispatus DSM25988 were found to efficiently bind to fibronectin (Figure 3). DSM25988 (live cells as well as the heat-treated preparation) are able to bind to fibronectin as well as $S$. pyogenes, and that this binding is stable after three wash cycles.

Based on analysis of different Lactobacillus strains preselected for their ability to co-aggregate with S. pyogenes, L. crispatus DSM25988 (strain 3 in Figure 4) was selected for further analysis as it is most likely to exhibit a good exclusion activity against S. pyogenes on fibronectin binding (Figure 4).

Competitive exclusion experiments also showed that a heat-treated lyophilised preparation of $L$. crispatus reduced binding of S. pyogenes DSM20565 to fibronectin reliably and at a comparable efficiency as the viable cell preparation. The competitive exclusion activity increases with the number of heat-treated lyophilised L. crispatus cells used (Figure 5), and degree of exclusion can reach up to $80 \%$ under the conditions used.

\section{Lactobacillus crispatus DSM 25988 neutralises SARS- CoV2 virus in a competitive binding assay}

As both bacterial and viral interactions with human epithelial cells predominantly occur via competitive binding to surface structures/receptors or direct fibronectin binding, we tested if $L$. crispatus DSM25988 exhibit activity in neutralise the SARS-CoV2 spike protein in a competitive binding experiment. Using a recombinant rVSV-Sars-CoV2 mutant and Vero cells, heat-treated $L$. crispatus cells were

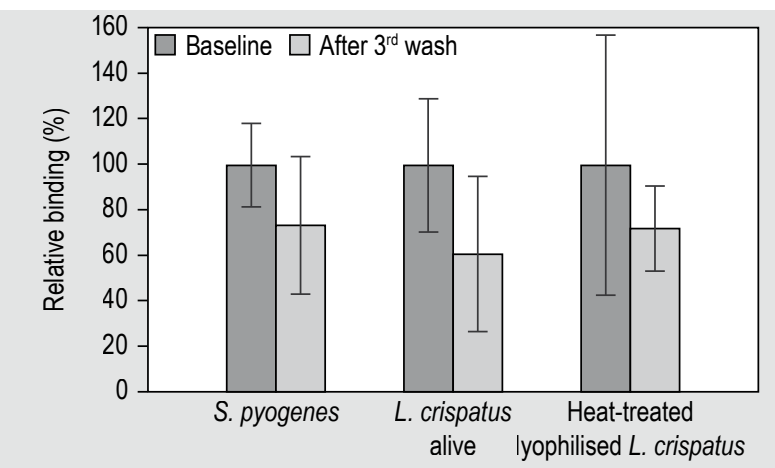

Figure 3. Efficiency of binding of Lactobacillus crispatus DSM25988 and Streptococcus pyogenes to fibronectin coated plates. Fibronectin binding is detected for $L$. crispatus DSM25988 and heat-treated lyophilised L. crispatus DSM25988 to a similar level as for $S$. pyogenes. S. pyogenes and $L$. crispatus were stained with CFDA SE. Both baseline fluorescence (dark grey columns, showing the total fluorescence of the stained sample bound and non-bound) and fluorescence after the three washing steps (light grey columns, showing the bound cells after removal of non-binding cells) were measured. Results show the data from 8 datasets.

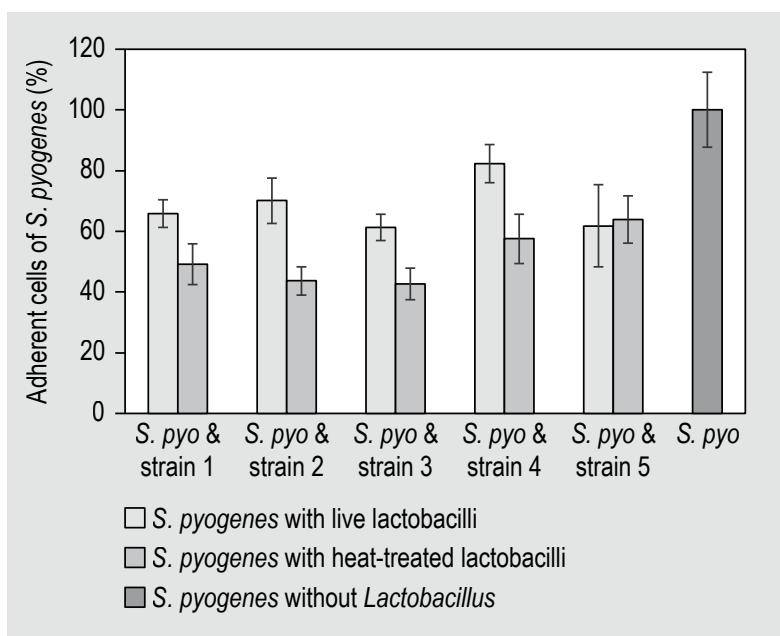

Figure 4. Lactobacillus cells reduce binding to fibronectin in a co-incubation-assay of Lactobacillus strains and Streptococcus pyogenes DSM20565. The diagram shows the percentage of adherent $S$. pyogenes cells in co-incubation with selected living and heat-treated Lactobacillus strains. The control shows S. pyogenes binding alone. Living, as well as heat-treated lactobacilli reduce the amount of $S$. pyogenes binding to fibronectin. Results show the data from 8 datasets.

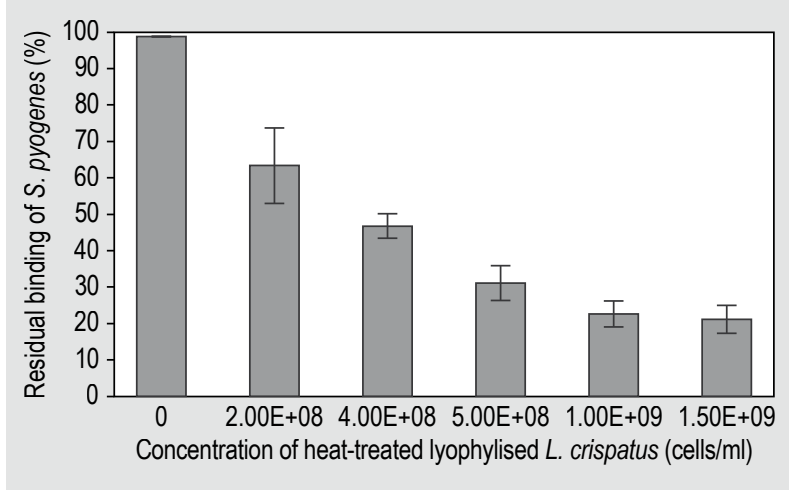

Figure 5. Competitive binding of Streptococcus pyogenes to fibronectin in a co-incubation assay with heat-treated lyophilised Lactobacillus crispatus DSM25988 used at different concentrations. Data show concentration-dependent decrease in binding efficiency of $S$. pyogenes to fibronectin in a coincubation assay with $L$. crispatus DSM25988. Results show the data from 8 datasets.

co-incubated and exclusion of virus protein binding was detected by fluorescence (luciferase assay). Neutralisation of rVSV-Sars-CoV2 mutant occurred at low concentrations while no cytotoxicity (CC50) of the preparation was detected (Table 1). Also, when the other tested Lactobacillus strains showed an even better neutralisation rate, it has to be considered that the cytotoxicity was much higher for these strains. Both parameters have to be seen in a context for further applications. Based on these results, L. crispatus showed the most promising results for further applications. 
Table 1. Inhibition of rVSV-Sars-CoV2 mutant mean fluorescence signals in Vero cells by heat-treated lyophilised Lactobacillus crispatus DSM 25988 compared to non-related Lactobacillus strains (strains A and B). Results show data from 3 datasets.

$\begin{array}{lll}\text { Strain } & \begin{array}{l}\text { Concentration of } \\ \mathbf{5 0 \%} \text { neutralisation } \\ (\mathbf{m g} / \mathbf{m l})\end{array} & \begin{array}{l}\mathbf{5 0 \%} \text { cytotoxicity } \\ \text { concentration } \\ (\mathbf{m g} / \mathbf{m l})\end{array} \\ & 7.421 & 44.24 \\ \text { Heat-treated lyophilised } & & \\ \text { L. crispatus } & 5.946 & 9.614 \\ \text { Lactobacillus strain A } & 2.974 & 5.473 \\ \text { Lactobacillus strain B } & & \end{array}$

\section{Verification of co-aggregation activity of Lactobacillus crispatus after formulation into the medical device salvans lozenges}

The formulated heat-treated lyophilised $L$. crispatus DSM25988 in salvans lozenge was tested in vitro for coaggregation activity (Figure 6). Furthermore, the placebo lozenge without heat-treated lyophilised L. crispatus (salvans) was co-incubated with S. pyogenes to determine background co-aggregation. While the salvans lozenge alone showed a slight background reaction (auto-aggregation), all other negative controls did not show any co-aggregation at all. Co-incubation of the salvans lozenge and S. pyogenes however showed a clear co-aggregation and interaction between $S$. pyogenes and the medical device salvans lozenges. This co-aggregation was clearly stronger than the background reactions. Co-aggregation of the salvans lozenge with the $S$. pyogenes is maintained after formulation.

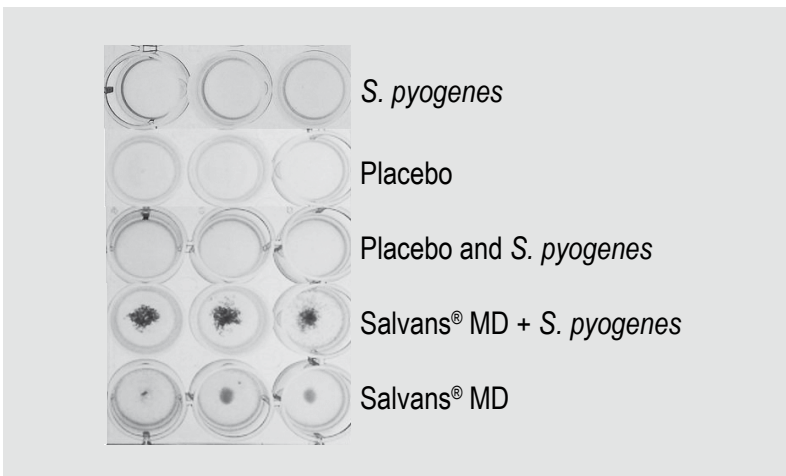

Figure 6. Co-aggregation of Streptococcus pyogenes and Lactobacillus crispatus in formulated salvans medical device (MD) lozenge. A lozenge without $L$. crispatus (placebo) and salvans lozenge were investigated in co-aggregation assays. As negative controls S. pyogenes alone, placebo alone or salvans lozenge alone were used. A slight background reaction (auto-aggregation) of salvans lozenge is visible, but can be neglected in comparison to the co-aggregation of salvans lozenge and S. pyogenes.

\section{Salvans lozenges user study}

Subjects from a customer database who had previously given consent to receive information about new products, were contacted and informed about heat-treated lyophilised L. crispatus containing salvans throat lozenges and were asked to participate in a post-market user study with the certified medical device class I. In total, 147 subjects with a history of signs and symptoms or diagnoses indicating multiple bacterial throat infections during the last 5 years consented to participate in the prospective, uncontrolled patient reported outcome study. Each study subject was provided with one package of salvans throat lozenges and the link to electronic baseline and follow-up questionnaires, respectively. 86 subjects (58.5\%) with an acute episode of throat irritation reported the baseline dataset (intentionto-treat dataset). Of these, 44 subjects (51\%) also reported follow-up data (per protocol dataset).

This study is the first to evaluate the impact of $L$. crispatus DSM25988 formulated in salvans lozenges on throat infection symptoms. Details of the study population and the baseline characteristics are given in Supplementary Table S1. The overall symptom rating and the results on the subcategories are summarised in Table 2 and in Supplementary Table S2.

The overall VAS rating significantly decreases during the study and highlights an overall improvement. The largest decrease was seen in the swallowing pain, agonizing throat pain, dry throat and scratchy sensation. No side effects were reported in the study group.

Table 2. Longitudinal reporting of symptom severity.

\begin{tabular}{|c|c|c|}
\hline & Baseline $(n=44)$ & $\begin{array}{l}\text { Follow-up } \\
(n=44)\end{array}$ \\
\hline Reported treatment duration (days) & - & $8.35(0,17)$ \\
\hline \multicolumn{3}{|l|}{ VAS symptom severity scoring ${ }^{1}$} \\
\hline 'agonising throat pain' & $4.82(0,9)$ & $1.36(0,8)$ \\
\hline 'burning' & $3.33(0,8)$ & $0.73(0,7)$ \\
\hline 'swallowing pain' & $4.38(0,9)$ & $1.09(0,8)$ \\
\hline 'dry throat' & $5.67(0,10)$ & $1.31(0,8)$ \\
\hline 'hoarseness' & $4.36(0,10)$ & $1.36(0,7)$ \\
\hline 'scratchy sensation' & $5.60(0,10)$ & $0.87(0,5)$ \\
\hline 'lump in the throat' & $2.56(0,10)$ & $0.76(0,7)$ \\
\hline 'inflamed throat' & $3.31(0,10)$ & $0.91(0,8)$ \\
\hline 'swollen throat' & $1.71(0,9)$ & $0.69(0,9)$ \\
\hline 'tightened feeling' & $1.84(0,9)$ & $0.60(0,7)$ \\
\hline
\end{tabular}




\section{Discussion and conclusions}

The main goal of this study was to identify and characterise a Lactobacillus strain that interacts with and co-aggregates S. pyogenes, the bacterial causative agent of throat infections, and to develop a suitable application device. We showed that selected Lactobacillus strains prevent adhesion and invasion of S. pyogenes to human epithelial pharyngeal cells. We identified strain L. crispatus DSM25988 as the best candidate to prevent $S$. pyogenes from binding to and invading human cells and as bioactive agent for the medical device as suitable application form.

Lactobacillus strains have long been used in food and dairy products. Many of those exhibit probiotic properties when applied as live cells and at adequate quantities and are proposed to be beneficial to human health, their main site of activity being the gastrointestinal tract (Silva et al., 2020; Walter 2008). Lactobacilli with beneficial effects occur naturally in the human body and include strains of the species Lactococcus lactis, Lacticaseibacillus paracase $i$ or L. crispatus (Abramov et al., 2014; Pasolli et al., 2020). When exploring and developing a novel strain for use in humans, several aspects have to be considered, among those being safety on the one hand and clear mode of action on the other hand (Doron and Snydman, 2015; FAO/WHO, 2002; Melo Pereira et al., 2018).

The safety characteristics are the strain's origin (healthy human microbiota), pathogenicity, and antibiotic resistance. L. crispatus is a naturally occurring Lactobacillus strain in the human body and is generally recognised as safe (GRAS-status). The genome analysis of $L$. crispatus DSM25988 showed that no antibiotic resistance genes or virulence factors are present, which is an additional safety aspect. Cytotoxicity has proved low. Activities of probiotic strains include epithelium adhesion ability, pathogen coaggregation and antimicrobial activity. The coaggregation properties of a probiotic strain with potential pathogens can be used as a screening marker for the selection of the probiotic strain for administration to humans (Melo Pereira et al., 2018). Coaggregation enables the pathogen agglomeration with the probiotic cells, which results in the elimination of pathogen through natural body functions (faeces, saliva).

The activity of $L$. crispatus DSM25988 is partly based on its efficacy in binding to $S$. pyogenes cells. Co-aggregation has previously been described as a potent mode of action for bacterial interactions with the aim to remove single species from surfaces or bacterial communities. Recent examples include the co-aggregation of Helicobacter pylori in the gastric system, Candida spp. in the vaginal cavity or Streptococcus mutans in the oral cavity (Buckley et al., 2018; Carmo et al., 2016; Holz et al., 2013, 2015). Co-aggregation which might also lead to 'masking' the microbial cell surface binding molecules and sterically hinder binding of the pathogen to epithelial surface molecules may lead to detachment of target cells from the biofilm or from epithelial tissue, thus reducing inflammatory signals or biofilm stability. Additionally, the larger co-aggregate can more easily be removed from the oral cavity or other surfaces than single microbial cells.

L. crispatus DSM25988 also exhibits a binding activity to human cells, among others to cell surface structures like fibronectin. We propose that L. crispatus DSM25988 is able to directly interact with $S$. pyogenes and to bind to fibronectin which might exclude $S$. pyogenes from binding to epithelial cells. Interestingly, this latter feature makes L. crispatus DSM25988 a potential bioactive agent against viral particles that rely on fibronectin binding for cell invasion. We used SARS-CoV2 protein as a model system in this study to demonstrate this effect. More viruses, such as influenza virus, use fibronectin as binding partner to invade human cells (Greco et al., 2002; Leung et al., 2012). In vivo co-aggregation of group A streptococcus (GAS) streptococci, masking their surface sites ordinarily available for binding to epithelial cells, is suggested to reduce acute pharyngitis/tonsillitis risk by fostering clearance of planktonic GAS streptococci from the oral cavity by forming larger aggregate which are flushed from the oral cavity and throat by batural saliva flow. Manna et al. (2020) recently identified probiotic-derived lipopeptides as potential inactivating molecule for SARS-CoV 2 Spike protein and its human receptor ACE2. They hypothesised an immunomodulatory role of such metabiotic compounds. We propose that interaction of L. crispatus DSM25988 with fibronectin might be a therapeutic mechanism to exclude viruses from infection in human tissues as has previously been shown in the vaginal cavity (Abramov et al., 2014; He et al., 2020; Ojala et al., 2014).

Different approaches had been used to identify potential aggregation-involved structures (Kojic et al., 2011; Miljkovic et al., 2015; Schachtsiek et al., 2004; Younes et al., 2012). Recent reviews summarise the diverse surface molecules found on Lactobacillus cells that are part of the pathogen and epithelium interactions (Muscariello et al., 2020; Sengupta et al., 2013). These include aggregation promoting factors in Lactiplantibacillus plantarum, L. acidophilus and Lactobacillus gasseri (Goh and Klaenhammer 2010). Which of those molecules are responsible for the observed activities in the Lactobacillus strain studied here, is part of our ongoing research.

We furthermore described the formulation of heat-treated Lactobacillus crispatus into lozenges (certified medical device class $1, \mathrm{MDD}$ ) in a user study. S. pyogenes (also known as group A $\beta$-haemolytic Streptococcus or GABHS) is the most common bacterial cause of acute sore throat. Antibiotics are still the most often prescribed therapy for 
acute sore throat and are often unnecessary and ineffective in this setting, contributing to the growing problem of antibiotic resistance. Lozenges containing antiseptics have a broad range antibacterial activity which damages the oral microbiota as a severe side effect. Addressing microbiota management as an alternative to antimicrobial drugs has been proposed earlier and might be a valid paradigm change in treating infections (Humphreys and McBain 2019). Using inactivated, i.e. heat-treated cells of biologically active Lactobacillus cells is emerging as a new approach to deliver biological activity via metabolites or cell surface structures (Geraldo et al., 2020; Shenderov et al., 2020). Inactivated probiotics have been described to enhance host immunity or to protect against pathogens (Jeong et al., 2020; Jia et al., 2019).

A specific and targeted approach might prove a noteworthy alternative that helps to reduce the use of antibiotics and broad-spectrum antimicrobials in over the counter lozenges for sore throat application.

\section{Supplementary material}

Supplementary material can be found online at https://doi. org/10.3920/BM2021.0103.

Table S1. Baseline characteristics of salvans throat lozenges user group (intention to treat and per protocol cohort).

Table S2. Longitudinal reporting of treatment satisfaction and product safety at baseline and follow-up.

\section{Conflict of interest}

CL holds shares of Belano medical AG.

\section{References}

Abramov, V., Khlebnikov,V., Kosarev, I., Bairamova, G., Vasilenko, R., Suzina, N., Machulin, A., Sakulin, V., Kulikova, N., Vasilenko, N., Karlyshev, A., Uversky, V., Chikindas, M.L. and Melnikov, V., 2014. Probiotic properties of Lactobacillus crispatus 2,029: homeostatic interaction with cervicovaginal epithelial cells and antagonistic activity to genitourinary pathogens. Probiotics and Antimicrobial Proteins 6: 165-176. https://doi.org/10.1007/s12602-014-9164-4.

Andreoletti, O., Budka, H., Buncic, S., Colin, P., Collins, J.D., De Koeijer, A., Griffin, J., Havelaar, A., Hope, J., Klein, G., Kruse, H., Magnino, S., López, A.M., McLauchlin, J., Nguyen-Thé, C., Noeckler, K., Noerrung, B., Prieto Maradona, M., Roberts, T., Vågsholm, I. and Vanopdenbosch, E., 2008. Scientific Opinion of the Panel on Biological Hazards on a request from EFSA on the maintenance of the QPS list of microorganisms intentionally added to food or feed. EFSA Journal 6: 923. https://doi.org/10.2903/j.efsa.2008.923
Antikainen, J., Anton, L., Sillanpää, J. and Korhonen, T.K., 2002. Domains in the S-layer protein CbsA of Lactobacillus crispatus involved in adherence to collagens, laminin and lipoteichoic acids and in self-assembly. Molecular Microbiology 46: 381-394. https:// doi.org/10.1046/j.1365-2958.2002.03180.x

Beganović, J., Kos, B., Leboš Pavunc, A., Uroić, K., Jokić, M. and Šušković, J., 2014. Traditionally produced sauerkraut as source of autochthonous functional starter cultures. Microbiological Research 169: 623-632. https://doi.org/10.1016/j.micres.2013.09.015

Buckley, M., Lacey, S., Doolan, A., Goodbody, E. and Seamans, K., 2018. The effect of Lactobacillus reuteri supplementation in Helicobacter pylori infection: a placebo-controlled, single-blind study. BMC Nutrition 4: 48. https://doi.org/10.1186/s40795-018-0257-4.

Carapetis, J.R., Steer, A.C., Mulholland, E.K. and Weber, M., 2005. The global burden of group A streptococcal diseases. The Lancet Infectious Diseases 5: 685-694. https://doi.org/10.1016/S14733099(05)70267-X

Carmo, M.S., Noronha, F.M.F., Arruda, M.O., Costa, Ê.P.S., Bomfim, M.R.Q., Monteiro, A.S., Ferro, T.A.F., Fernandes, E.S., Girón, J.A. and Monteiro-Neto, V., 2016. Lactobacillus fermentum ATCC 23271 displays in vitro inhibitory activities against Candida spp. Frontiers in Microbiology 7: 1722. https://doi.org/10.3389/fmicb.2016.01722

Ceri, H., Olson, M.E., Stremick, C., Read, R.R., Morck, D. and Buret, A., 1999. The Calgary Biofilm Device: new technology for rapid determination of antibiotic susceptibilities of bacterial biofilms. Journal of Clinical Microbiology 37: 1771-1776. https://doi. org/10.1128/JCM.37.6.1771-1776.1999

Chen, X., Xu, J., Shuai, J, Chen, J, Zhang, Z. and Fang, W., 2007. The S-layer proteins of Lactobacillus crispatus strain ZJ001 is responsible for competitive exclusion against Escherichia coli O157:H7 and Salmonella Typhimurium. International Journal of Food Microbiology 115: 307-312. https://doi.org/10.1016/j. ijfoodmicro.2006.11.007

Courtney, H.S., Ofek, I., Simpson, W.A., Hasty, D.L., Beachey, E.H., 1986. Binding of Streptococcus pyogenes to soluble and insoluble fibronectin. Infection and Immunity 53: 454-459. https://doi. org/10.1128/iai.53.3.454-459.1986

Danzmann, L., Gastmeier, P., Schwab, F., and Vonberg, R.P., 2013. Health care workers causing large nosocomial outbreaks: a systematic review. BMC Infectious Diseases 13: 98. https://doi. org/10.1186/1471-2334-13-98

De Man, J.C., Rogosa, M. and Sharpe, M.E., 1960. A medium for the cultivation of lactobacilli. Journal of Applied Bacteriology 23: 130135. https://doi.org/10.1111/j.1365-2672.1960.tb00188.x

Doron, S. and Snydman, D.R., 2015. Risk and safety of probiotics. Clinical Infectious Diseases 60: 129-134. https://doi.org/10.1093/ cid/civ085

Ellison, A.J., Dempwolff, F., Kearns, D.B. and Raines, R.T., 2020. Role for cell-surface collagen of Streptococcus pyogenes in infections. ACS Infectious Diseases 10: 1836-1843. https://doi.org/10.1021/ acsinfecdis.0c00073

Ellner, P.D., Stoessel, C.J., Drakeford, E. and Vasi, F., 1966. A new culture medium for medical bacteriology. American Journal of Clinical Pathology 45: 502-504. https://doi.org/10.1093/ajcp/45.4_ts.502 
European Commission (EC), 2016. Regulation (EU) 2016/679 of the European Parliament and of the Council of 27 April 2016 on the protection of natural persons with regard to the processing of personal data and on the free movement of such data, and repealing Directive 95/46/EC (General Data Protection Regulation). Official Journal of the European Union L 119: 1-88.

Food and Agriculture Organization of the United Nations/World Health Organization (FAO/WHO), 2002. Guidelines for the evaluation of probiotics in food. Report of a Joint FAO/WHO working group on drafting guidelines for the evaluation of probiotics in food. Available at: http://tinyurl.com/zdbrkeg.

Geraldo, B.M.C., Batalha, M.N., Milhan, N.V.M., Rossoni, R.D., Scorzoni, L. and Anbinder, AL., 2020. Heat-killed Lactobacillus reuteri and cell-free culture supernatant have similar effects to viable probiotics during interaction with Porphyromonas gingivalis. Journal of Periodontal Research 55: 215-220. https://doi.org/10.1111/ jre.12704

Goh, Y.J. and Klaenhammer, T.R., 2010. Functional roles of aggregationpromoting-like factor in stress tolerance and adherence of Lactobacillus acidophilus NCFM. Applied and Environmental Microbiology 76: 5005-5012. https://doi.org/10.1128/AEM.00030-10 Greco, G., Pal, S., Pasqualini R. and Schnapp, L.M., 2002. Matrix fibronectin increases HIV stability and infectivity. Journal of Immunology 168: 5722-5729. https://doi.org/10.4049/ jimmunol.168.11.5722

Harrison, J.J., Stremick, C.A., Turner, R.J., Allan, N.D., Olson, M.E. and Ceri H., 2010. Microtiter susceptibility testing of microbes growing on peg lids: a miniaturized biofilm model for high-throughput screening. Nature Protocols 5: 1236-1254. https://doi.org/10.1038/ nprot.2010.71

He, Y., Niu, X., Wang, B., Na, R., Xiao, B. and Yang, H., 2020. Evaluation of the inhibitory effects of Lactobacillus gasseri and Lactobacillus crispatus on the adhesion of seven common lower genital tract infection-causing pathogens to vaginal epithelial cells. Frontiers in Medicine 7: 284. https://doi.org/10.3389/fmed.2020.00284

Henri-Dubernet, S., Desmasures, N. and Guéguen, M., 2008. Diversity and dynamics of lactobacilli populations during ripening of RDO Camembert cheese. Canadian Journal of Microbiology 54: 218-228. https://doi.org/10.1139/W07-137

Holz, C., Alexander, C., Balcke, C., Moré, M., Auinger, A., Bauer, M., Junker, L., Grünwald, J., Lang, C. and Pompejus, M., 2013. Lactobacillus paracasei DSMZ16671 reduces mutans streptococci: a short-term pilot study. Probiotics and Antimicrobial Proteins 5: 259-263. https://doi.org/10.1007/s12602-013-9148-9

Holz, C., Busjahn, A., Mehling, H., Arya, S., Boettner, M., Habibi, H. and Lang, C., 2015. Significant reduction in Helicobacter pylori load in humans with non-viable Lactobacillus reuteri DSM17648: a pilot study. Probiotics and Antimicrobial Proteins 7: 91-100. https://doi. org/10.1007/s12602-014-9181-3

Horie, M., Ishiyama, A., Fujihira-Ueki, Y., Sillanpää, J., Korhonen, T.K. and Toba, T., 2002. Inhibition of the adherence of Escherichia coli strains to basement membrane by Lactobacillus crispatus expressing an S-layer. Journal of Applied Microbiology 92: 396-403. https:// doi.org/10.104.6/j.1365-2672.2002.01539.x
Humphreys, G. and McBain, A., 2019. Antagonistic effects of Streptococcus and Lactobacillus probiotics in pharyngeal biofilms. Letters in Applied Microbiology 68: 303-312. https:// doi.org/10.1111/lam.13133

Jeong, M., Kim, J.H., Lee, J.S., Kang, S.D., Shim, S., Jung, M.Y., Yang, H., Byun, S. and Lee, K.W., 2020. Heat-killed Lactobacillus brevis enhances phagocytic activity and generates immunestimulatory effects through activating the TAK1 pathway. Journal of Microbiology and Biotechnology 30: 1395-1403. https://doi. org/10.4014/jmb.2002.02004

Jia, H., Ren, S. and Wang, X., 2019. Heat-killed probiotic regulates the body's regulatory immunity to attenuate subsequent experimental autoimmune arthritis. Immunology Letters 216: 89-96. https://doi. org/10.1016/j.imlet.2019.10.009

Kačániová, M., Hleba, L., Pochop, J., Kádasi-Horáková, M., Fikselová, M. and Rovná, K., 2012. Determination of wine microbiota using classical method, polymerase chain method and step one realtime PCR during fermentation process. Journal of Environmental Science and Health Part B 47: 571-578. https://doi.org/10.1080/0 3601234.2012.665750

Kim, S.Y., Choi, D.J., and Chung J., 2015. Antibiotic treatment for Helicobacter pylori: is the end coming? World Journal of Gastrointestinal Pharmacology and Therapeutics 6: 183-198. https:// doi.org/10.4292/wjgpt.v6.i4.183

Kojic, M., Jovcic, B., Strahinic, I., Begovic, J., Lozo, J., Veljovic, K. and Topisirovic, L., 2011. Cloning and expression of a novel lactococcal aggregation factor from Lactococcus lactis subsp. lactis BGKP1. BMC Microbiology 11: 265. https://doi.org/10.1186/1471-218011-265

Lang, C., Böttner, M., Holz, C., Veen, M., Ryser, M., Reindl, A., Pompejus, M. and Tanzer, J.M., 2010. Specific Lactobacillus/mutans Streptococcus co-aggregation. Journal of Dental Research 89: 175179. https://doi.org/10.1177/0022034509356246

Langdon, A., Crook, N. and Dantas, G., 2016. The effects of antibiotics on the microbiome throughout development and alternative approaches for therapeutic modulation. Genome Medicine 8: 39. https://doi.org/10.1186/s13073-016-0294-Z

Leung, H.S.Y., Li, O.T.W., Chan, R.W.Y., Chan, M.C.W., Nicholls, J.M. and Poon, L.L.M., 2012 Entry of influenza A virus with a $\alpha 2,6$-linked sialic acid binding preference requires host fibronectin. Journal of Virology 86: 10704-10713. https://doi.org/10.1128/JVI.01166-12

Lindbaek, M., Høiby, E.A., Lermark, G., Steinsholt, I.M. and Hjortdahl, P., 2004. Predictors for spread of clinical group A streptococcal tonsillitis within the household. Scandinavian Journal of Primary Health Care 22: 239-243. https://doi. org/10.1080/02813430410006729

Manna, S., Chowdhury, T., Chakraborty, R. and Mandal, S.M., 2020. Probiotics-derived peptides and their immunomodulatory molecules can play a preventive role against viral diseases including COVID-19. Probiotics and Antimicrobial Proteins 23: 1-13. https:// doi.org/10.1007/s12602-020-09727-7

Melo Pereira, G., Oliveira Coelho, B., Magalhaes, A.I., Thomaz-Socol, V., and Sokol, C.R., 2018. How to select a probiotic? A review and update of methods and criteria. Biotechnology Advances 36: 20602076. https://doi.org/10.1016/j.biotechadv.2018.09.003 
Meroth, C.B., Walter, J., Hertel, C., Brandt, M.J., and Hammes, W.P., 2003. Monitoring the bacterial population dynamics in sourdough fermentation processes by using PCR-denaturing gradient gel electrophoresis. Applied and Environmental Microbiology 69: 475-482. https://doi.org/10.1128/AEM.69.1.475-482.2003

Miljkovic M., Strahinic I., Tolinacki M., Zivkovic M., Kojic S., Golic N., and Kojic M., 2015. AggLb is the largest cell-aggregation factor from Lactobacillus paracasei subsp. paracasei BGNJ1-64, functions in collagen adhesion, and pathogen exclusion in vitro. PLoS ONE 10: e0126387. https://doi.org/10.1371/journal.pone.0126387

Mousavi, E., Makvandi, M., Teimoori, A., Ataei, A, Ghafari, S. and Samarbaf-Zadeh, A., 2018. Antiviral effects of Lactobacillus crispatus against HSV-2 in mammalian cell lines. Journal of the Chinese Medical Association 81: 262-267. https://doi.org/10.1016/j. jcma.2017.07.010

Muscariello, L., De Siena, B. and Marasco, R., 2020. Lactobacillus cell surface proteins involved in interaction with mucus and extracellular matrix components. Current Microbiology 77: 3831-3841. https:// doi.org/10.1007/s00284-020-02243-5

Ngugi, B.M., Hemmerling, A., Bukusi, E.A., Kikuvi,. G, Gikunju, J., Shiboski, S., Fredricks, D.N. and Cohen, C.R., 2011. Effects of BVassociated bacteria and sexual intercourse on vaginal colonization with the probiotic Lactobacillus crispatus CTV-05. Sexually Transmitted Diseases 38: 1020-1027. https://doi.org/10.1097/ OLQ.0b013e3182267ac4

Ojala, T., Kankainen, M., Castro, J., Cerca, N., Edelman, S., WesterlundWikström, B., Paulin, L., Holm, L. and Auvinen, P., 2014. Comparative genomics of Lactobacillus crispatus suggests novel mechanisms for the competitive exclusion of Gardnerella vaginalis. BMC Genomics 15: 1070. https://doi.org/10.1186/1471-2164-15-1070

Parolin, C., Frisco, G., Foschi, C., Giordani, B., Salvo, M., Vitali, B., Marangoni, A. and Calonghi, N., 2018. Lactobacillus crispatus BC5 interferes with Chlamydia trachomatis infectivity through integrin modulation in cervical cells. Frontiers in Microbiology 9: 1-9. https://doi.org/10.3389/fmicb.2018.02630

Pasolli, E., De Filippis, F., Mauriello, I.E., Cumbo, F., Walsh, A.M., Leech, J., Cotter, P.D., Segata, N. and Ercolini, D., 2020. Large-scale genome-wide analysis links lactic acid bacteria from food with the gut microbiome. Nature Communications 11: 2610. https://doi. org/10.1038/s41467-020-16438-8

Plant, L., Sundqvist, J., Zughaier, S., Lövkvist, L., Stephens, D.S. and Jonsson, A., 2006. Lipooligosaccharide structure contributes to multiple steps in the virulence of Neisseria meningitidis. Infection and Immunity 74: 1360-1367. https://doi.org/10.1128/IAI.74.2.13601367.2006

Reid, G., and Friendship, R., 2002. Alternatives to antibiotic use: probiotics for the gut. Animal Biotechnology 13: 97-112. https:// doi.org/10.1081/abio-120005773

Rhoads, A. and Au, K.F., 2015. PacBio sequencing and its application. Genomics, Proteomics and Bioinformatics 13: 278-289. https://doi. org/10.1016/j.gpb.2015.08.002

Schachtsiek, M., Hammes, W.P. and Hertel, C., 2004. Characterization of Lactobacillus cornyformis DSM 20001T surface protein Cpf mediating coaggregation with and aggregation among pathogens. Applied and Environmental Microbiology 70: 7078-7085. https:// doi.org/10.1128/AEM.70.12.7078-7085.2004
Sengupta, R., Altermann, E., Anderson, R.C., McNabb, W.C., Moughan, P.J. and Roy, N.C., 2013. The role of cell surface architecture of Lactobacilli in host-microbe interactions in the gastrointestinal tract. Mediators of Inflammation 2013: 237921. https://doi. org $/ 10.1155 / 2013 / 237921$

Shenderov, B.A., Sinitsa, A.V., Zakharchenko, M. and Lang, C., 2020. Metabiotics. Springer Nature, Berlin, Germany.

Shulman, S.T., Bisno, A.B., Clegg, H.W., Gerber, M.A., Kaplan, E.L., Lee, G., Martin, J.M. and Van Beneden, C., 2012. Clinical practice guideline for the diagnosis and management of group $\mathrm{A}$ streptococcal pharyngitis. Clinical Infectious Diseases 55: e86-e102. https://doi.org/10.1093/cid/cis629

Silva, D.R., Sardi, J.O., Souza Pitanguic, N., Roque, S.M., Barbosa da Silva, A.C. and Rosalen, P.L., 2020. Probiotics as an alternative antimicrobial therapy: current reality and future directions. Journal of Functional Foods 73: 104080. https://doi.org/10.1016/j. jff.2020.104080

Stapleton, A.E., Au-Yeung, M., Hooton, T.M., Fredricks, D.N., Roberts, P.L., Czaja, C.A., Yarova-Yarovaya, Y., Fiedler, T., Cox, M. and Stamm, W.E., 2011. Randomized, placebo-controlled phase 2 trial of a Lactobacillus crispatus probiotic given intravaginally for prevention of recurrent urinary tract infection. Clinical Infectious Diseases 52: 1212-1217. https://doi.org/10.1093/cid/cir183

Stavropoulou, E. and Bezirtzoglou, E., 2020. Probiotics in medicine: a long debate. Frontiers in Immunology 11: 2192. https://doi. org/10.3389/fimmu.2020.02192

Stepanović, S., Vuković, D., Dakić, I., Savić, B. and Švabić-Vlahović, M., 2000. A modified microtiter-plate test for quantification of staphylococcal biofilm formation. Journal of Microbiological Methods 40: 175-179. https://doi.org/10.1016/S01677012(00)00122-6

Thors, V., Morales-Aza, B., Pidwill, G., Vipond, I., Muir, P. and Finn, A., 2016. Population density profiles of nasopharyngeal carriage of 5 bacterial species in pre-school children measured using quantitative PCR offer potential insights into the dynamics of transmission. Human Vaccines and Immunotherapeutics 12: 375-382. https:// doi.org/10.1080/21645515.2015.1090069

Van Beek, S. and Priest, F.G., 2000. Decarboxylation of substituted cinnamic acids by lactic acid bacteria isolated during malt whisky fermentation. Applied and Environmental Microbiology 66: 53225328. https://doi.org/10.1128/AEM.66.12.5322-5328.2000

Walker, M.J., Barnett, T.C., McArthur, J.D., Cole, J.N., Gillen, C.M., Henningham, A., Sriprakash, K.S., Sanderson-Smith, M.L. and Nizet, V., 2014. Disease manifestations and pathogenic mechanisms of group A Streptococcus. Clinical Microbiology Reviews 27: 264-301. https://doi.org/10.1128/CMR.00101-13

Walter, J., 2008. Ecological role of lactobacilli in the gastrointestinal tract: implications for fundamental and biomedical research. Applied and Environmental Microbiology 74: 4985-4996. https:// doi.org/10.1128/AEM.00753-08

Wang, H., Ma, Y., Li, R., Chen, X., Wan, L. and Zhao, W., 2019. Associations of cervicovaginal lactobacilli with high-risk human papillomavirus infection, cervical intraepithelial neoplasia, and cancer: a systematic review and meta-analysis. Journal of Infectious Diseases 220: 1243-1254. https://doi.org/10.1093/infdis/jiz325 


\section{A. Reiprich et al.}

You, I. and Kim, E.B., 2020. Genome-based species-specific primers for rapid identification of six species of Lactobacillus acidophilus group using multiplex PCR. PLoS ONE 15: e0230550. https://doi. org/10.1371/journal.pone.0230550
Younes, J.A., Van der Mei, H.C., Van den Heuvel, E., Busscher, H.J. and Reid, G., 2012. Adhesion forces and coaggregation between vaginal staphylococci and lactobacilli. PLoS ONE 7: e36917. https://doi. org/10.1371/journal.pone.0036917 